\title{
Dual Regimen with Stem Cell Antagonists and Differentiating Agents for Effective Chemotherapy
}

\author{
Achuta Kumar Guddati* \\ Massachusetts General Hospital, Harvard Medical School, Boston, USA
}

\begin{abstract}
Chemoresistance and radioresistance of various malignancies has been attributed to the presence of cancer stem cells. Elimination of these cancer stem cells has been postulated to help enhance the effectiveness of conventional chemotherapy by decreasing resistance to chemotherapy and radiotherapy. However identifying cancer stem cells and selectively targeting them has been a challenge due to the lack of unique markers. Interconversion of more differentiated cells to less differentiated cells may potentially confound this problem. A mathematical model which simulates stem cell homeostasis with such inbuilt probability of interconversion is presented here. Perturbing the system by introducing various agents which enhance differentiation leads to interesting results. Utilizing pure stem cell antagonists does not lead to elimination of the tumor cells due to dedifferentiation. Differentiation of specific subsets of cells also does not lead to elimination of cancer cells and in some situations may paradoxically drive tumor growth in a robust manner. The model predicts that a dual regimen consisting of a stem cell antagonist and a differentiating agent which acts on multiple cell populations is effective in decelerating the tumor growth and in eliminating the tumor cells. This model provides a theoretical framework for the applicability of such a dual regimen in cancer treatment.
\end{abstract}

Keywords: Dual regimen; Differentiating; Chemotherapy; Stem cell antagonists

\section{Introduction}

Tumors have been observed to be heterogeneous in their constituency [1]. The relative abundance of differentiated cells and undifferentiated cells in a tumor, especially after chemotherapy has been observed to correlate with prognosis and recurrence [2]. It has been increasingly recognized that tumor growth is driven by cancer stem cells (CSCs) [3]. Cells with cancer stem cell properties have been characterized in multiple malignancies including skin, brain, breast, pancreas, prostate, colon and urinary bladder [4-10]. Eliminating cancer stem cells has been postulated to be an effective method to cure cancer; however a panel of markers that uniquely identify cancer stem cells is currently not available [11]. It has been observed in acute myeloid leukemia that populations at different layers of the differentiation hierarchy may interconvert and recapitulate the heterogeneity of the resultant tumor cells $[12,13]$. The interconversion has an important bearing on overall cancer treatment as it implies that primitive cells are replenished by dedifferentiation of more differentiated cells with less proliferative capacity. It has been shown that subsets of tumorigenic cell populations in colon and pancreatic cancer may contribute to metastasis $[14,15]$. Therefore, eliminating cancer stem cells and the cells which may potentially dedifferentiate into cancer stem cells may not only help decelerate the growth of the primary tumor but may also decrease the chances of metastasis. A mathematical model is constructed which shows that a combination of stem cell antagonists and differentiating agents is superior to either of the regimens alone. The hypothesis being investigated is that stem cell antagonists and differentiating agents when applied together work synergistically to prevent repletion of stem cells by dedifferentiation and hence have a greater probability of eliminating the tumor cells. Preliminary simulation data is presented to support the hypothesis. Various scenarios have been simulated to demonstrate the effect of differentiation on different layers of the stem cell hierarchy.

\section{Methods}

A mathematical model with four layers has been constructed to reflect the hierarchal arrangement of cancer stem cells, progenitor cells and, transit-amplifying cells and differentiated cells. Cancer stem cells are depicted in the apex in Figure 1A where they differentiate to form progenitor cells. These divisions may include both self-renewal and differentiation into progenitor cells. The cascade of differentiation continues from the progenitor cells to transit amplifying cells and finally to differentiated cells. It is assumed that the cells attain more differentiated phenotype as they progress downwards in the cascade accompanied by a simultaneous loss of proliferative potential. It should be noted that cells which reside in the fourth layer are capable of dedifferentiation and they exit the cascade to form terminally differentiated cells which have a limited life span and as such are not depicted in this model. It is assumed in this model that the terminally differentiated cells do not have the ability to dedifferentiate and therefore do not influence the homeostasis of the stem cell compartment. This model is similar in concept to the phase-space model of hematopoietic stem cells and parameters to construct the model have been adapted from it [16]. Repletion of different compartments is enabled by allowing dedifferentiation across all the layers of the cascade. The model is initially seeded with cancer stem cells and the model achieves homeostasis gradually and approximately after 80 cell divisions. The effect on cell numbers is depicted under various conditions of perturbation of elimination and differentiation. This model specifically intends to depict the effect of dedifferentiation on homeostasis of cells

*Corresponding author: Achuta Kumar Guddati, MD, PhD, Massachusetts General Hospital, 50 Fruit Street, Boston, MA, USA, Tel: 312-404-8928; E-mail: aguddati@partners.org

Received November 12, 2012; Accepted December 20, 2012; Published December 22, 2012

Citation: Achuta Kumar G (2012) Dual Regimen with Stem Cell Antagonists and Differentiating Agents for Effective Chemotherapy. J Stem Cell Res Ther S7:008. doi:10.4172/2157-7633.S7-008

Copyright: (c) 2012 Achuta Kumar G. This is an open-access article distributed under the terms of the Creative Commons Attribution License, which permits unrestricted use, distribution, and reproduction in any medium, provided the original author and source are credited. 
in the various layers of the stem cell compartment. The parameters used to depict the stem cell model are provided in Table 1. The stem cell antagonist is assumed to act with $50 \%$ efficiency and the results are depicted with introduction and withdrawal of the agent at specific time points. The model also assumes that dedifferentiation is initiated when the cell number in the stem cell layer falls below 400, progenitor population falls below 1000 and transit amplifying cell population falls below 2000. The model is simplistic in its approach and in its current form does not include other perturbations such as resistance to the applied agents, altered bioavailability of the applied agents to different cell layers and the effect of angiogenesis on the availability of the applied agents.

\section{Results}

The mathematical model presented here is unique in incorporating the possibility of dedifferentiation at all levels of the hierarchy to ensure homeostasis of the individual cell layers. The flow of cells starting from the stem cells to differentiated cells is depicted in Figure 1A. In this steady state, depletion of the stem cell layer by apoptosis and differentiation triggers dedifferentiation from the progenitor layer and replenishment of the stem cells. The bidirectional arrows depicted in Figure 1A represent the dedifferentiation ability of cells in all the layers and hence replenishment is possible in all the layers. Figure $1 \mathrm{~B}$ shows the establishment of a constant number of cells in each layer over an extended period of time. This represents the homeostatic state of the stem cell compartment after taking into account the processes of division, apoptosis, differentiation and dedifferentiation. The trigger for activation of the de-differentiation was set at $40 \%$ of the total stem cell population. The model serves to show the effect of antagonists and differentiating agents in different layers at different time points.

\section{Effect of stem cell antagonists}

Introducing a drug which directly acts on cancer stem cells and eliminates them and results in a decrease in the cell numbers across all the other layers as depicted in Figure 1C. It noticeably also results in the establishment of a new equilibrium. Therefore attempting to eliminate the cancer stem cell alone is not an optimal strategy. In Figure $1 \mathrm{C}$ the stem cell antagonist was introduced after 80 cell divisions and withdrawn after 250 cell divisions. The replenishment of the stem cell compartment by de-differentiation causes a robust increase in the tumor cells in all layers leading to the re-establishment of the steady state at its former level. This is representative of a complete recurrence.

\section{Effect of differentiating agents}

Introduction of a differentiating agent which specifically acts on progenitor cells is shown in Figure 2A. The agent is introduced after 80 cell divisions and causes a remarkable increase in the number of cells in the subsequent layers and a likely increase in the overall size of the tumor. This phenomenon is due to the high proliferative potential of cells in the cascade below that of progenitor cells. The depleted progenitor cells are well replenished by the stem cell compartment and differentiating the progenitor cell layer increases the supply of highly proliferative cells. This effect illustrates the danger involved in utilizing agents which specifically act on progenitor cells. Figure 2B illustrates the effect of a differentiating agent which specifically acts on transit amplifying cells. Notably, the effect is very similar to that of stem cell antagonists with the establishment of a new equilibrium at approximately the same level. Figure $2 \mathrm{C}$ depicts the effect of a differentiating agent which specifically acts on the last layer of the cascade; the differentiated cells. It specifically causes the efflux of cells

\begin{tabular}{|l|l|l|l|l|}
\hline & P Div & P Apop & P Diff & P Dediff \\
\hline Stem cells & 0.1 & 0 & 0.15 & 0 \\
\hline Progenitor cells & 0.2 & 0.02 & 0.22 & 0.05 \\
\hline TA cells & 0.4 & 0.05 & 0.37 & 0.05 \\
\hline Differentiated Cells & 0.6 & 0.088 & 0.6 & 0.05 \\
\hline
\end{tabular}

P Div: Probability of Division

P Apop: Probability of Apoptosis

P Diff: Probability of Differentiation

P Dediff: Probability of Dedifferentiation

Table 1: The probabilities of division, apoptosis, differentiation and dedifferentiation for various cell populations used in the construction of the model.

from this layer into a terminally differentiated layer of cells which do not have any dedifferentiation potential. These cells, although are a part of the tumor are assumed to have very limited life span and undergo apoptosis. Comparison of the effect of this agent with the other agents shows that the new equilibrium established is at a lower level. Figure $3 \mathrm{~A}$ illustrates the effect of a differentiating agent which acts on the progenitor, transit amplifying cells and the differentiated cells. The result is the establishment of a new equilibrium which is lower than that of all agents which act on individual layers of cells. However, the establishment of a new lower but ongoing equilibrium indicates that the tumor will continue to survive despite differentiation pressure on all layers below the stem cells in the differentiation cascade.

\section{Effect of the combination of stem cell antagonists and differentiating agents}

The effect of a combination of a stem cell antagonist and a differentiating agent which acts on progenitor cells, transit amplifying cells and differentiated cells is depicted in Figure 3B. Unlike, the effect of these agents when used individually, a combination does not result in a sustained new equilibrium but leads to a complete decline of the cell population in all the cell layers. This is representative of a complete remission. Withdrawal of the combination of these agents does not lead to a recurrence as the cells which could dedifferentiate and replenish the different compartments are depleted. This model suggests that in order to eliminate the possibility of recurrence, a combination of an agent to antagonize stem cells and a differentiating agent which acts on the rest of cascade is required.

\section{Discussion}

Cancer stem cells have been shown to be possible seats of resistance to radiation in brain and breast tumors $[17,18]$. However, these cells can be induced to differentiate as seen with the effect of bone morphogenetic protein 4 (BMP4) on glial stem cells and trans-retinoic acid in acute promyelocytic leukemia $[19,20]$. The mathematical model proposed here assumes that there is a hierarchy emanating from cancer stem cells but the existence and extent of this hierarchy may vary in different malignancies. In human AML, there are rare leukemogenic cells while in mouse acute lymphoblastic leukemia (ALL), more than half of the cell population is leukemogenic [21,22]. In melanoma, there is no evidence of the existence of such a hierarchy [23]. The bidirectional interconversion of cells between different layers in a stem cell differentiation cascade has been observed [24,25]. The frequency and rapidity of interconversion between tumorigenic and non-tumorigenic phenotype may provide an alternate pathway to the emergence of resistance besides known mechanisms such as de novo mutations. The mathematical model constructed here leads to some interesting and important predictions: 1 . Stem cell antagonists, even with a high efficiency may not necessarily lead to the eradication of the tumor. 2. Differentiating agents which act specifically on progenitor cells may 
Citation: Achuta Kumar G (2012) Dual Regimen with Stem Cell Antagonists and Differentiating Agents for Effective Chemotherapy. J Stem Cell Res

A

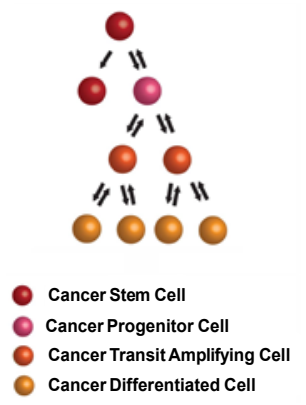

B

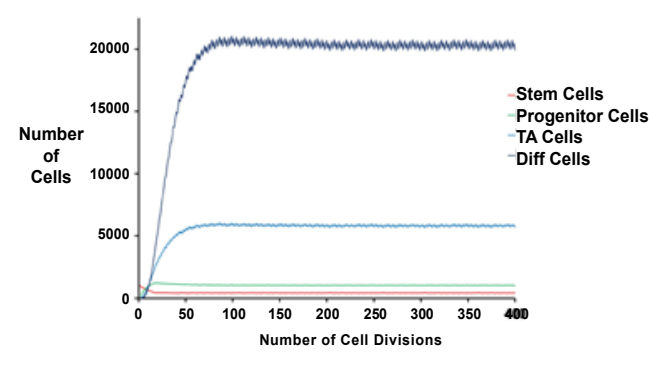

C

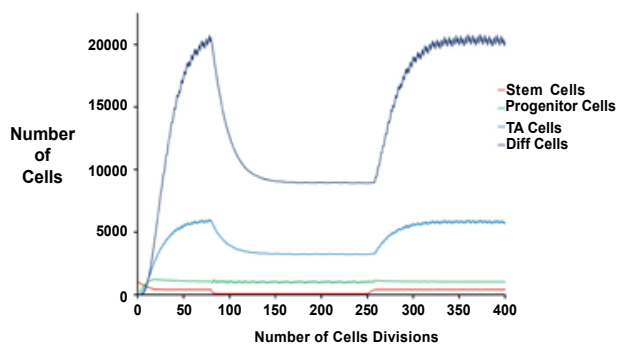

Figure 1: Panel A. The cancer stem cell compartment is depicted in four layers of cells with increasing differentiation and decreasing proliferative capacity. The bidirectional arrows represent dedifferentiation ability of these cells. Panel B. The establishment of a steady state in the stem cell compartment is noticeable after approximately 80 cell divisions. The compartment is initially seeded only with stem cells which divide, differentiate and establish a cascade with four different types of cells. Panel C. The effect of the introduction of a stem cell antagonist after 80 cell divisions results in an initial decline. Withdrawal of the agent after 250 cell divisions causes a re-establishment of the compartment which is representative of complete recurrence.

A

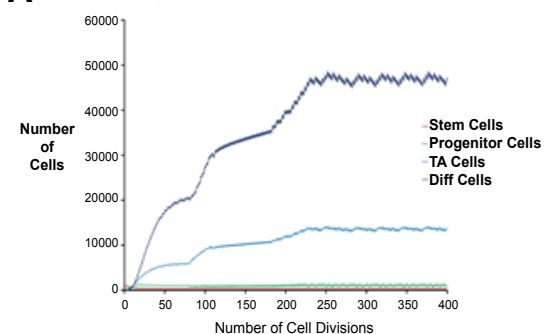

B

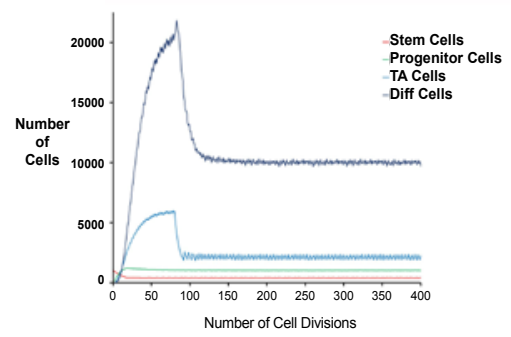

C

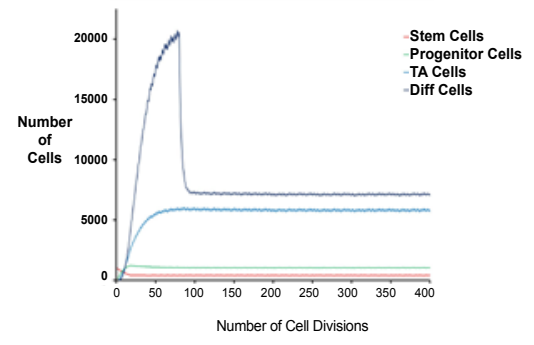

Figure 2: Panel A. Differentiating agent which acts on progenitors cells results in an increase in the overall number of cells and establishment of new equilibrium at a higher level. Panel B. Differentiating agent which acts on transit amplifying cells decreases the overall cell number but establishes am equilibrium at a level which is similar to that of stem cell antagonists. Panel C. Differentiating agent which acts on differentiated cells produces the most pronounced suppression on tumor cell population but nevertheless contributes to the formation a new but lower equilibrium.

A

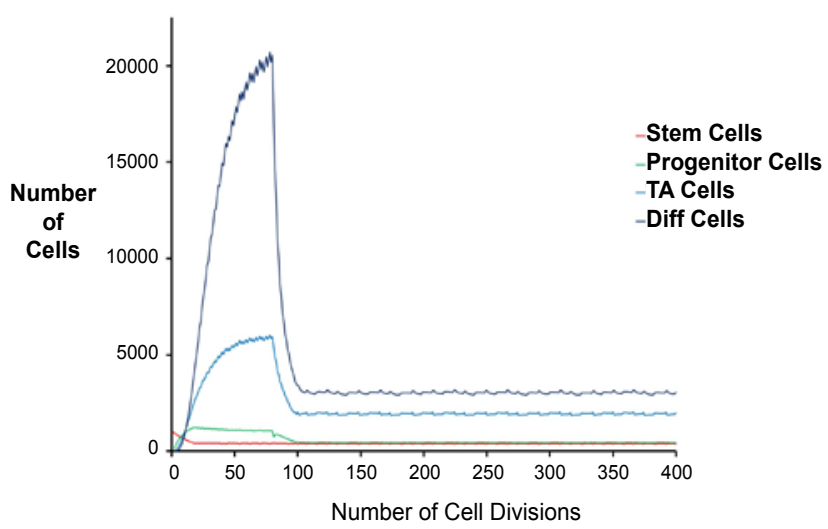

B

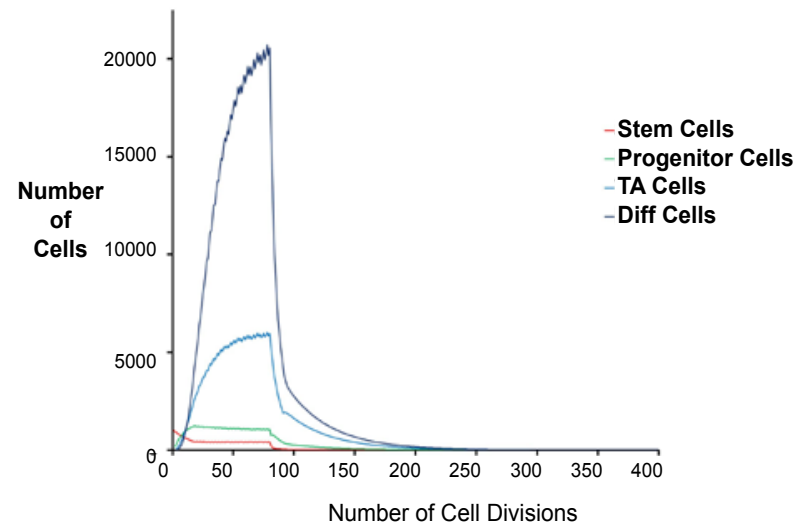

Figure 3: Panel A. Differentiating agent which acts on the progenitor cells, transit amplifying cells and the differentiated cells dramatically decreases the tumor cell population but does not lead to the elimination of cancer cells. Panel B. A dual regimen consisting of a combination of a stem cell antagonist and a differentiating agent which acts on the progenitor cells, transit amplifying cells and the pre-differentiated cells causes a rapid depletion of the cell population in all layers and complete resolution of the tumor. 
paradoxically increase the tumor mass by driving the cascade forward and by increasing the number of cells with high proliferative potential. 3. Differentiating agents which act on multiple cell populations have a greater effect in regressing the tumor but are not effective in eliminating the tumor cells. 4 . A combination of a stem cell antagonist and a differentiating agent which acts in multiple cell populations has a potent effect on regressing and more notably, eliminating the tumor cells. The model proposed in this chapter is simplistic and has several assumptions and drawbacks. The efficiency of the stem cell antagonist was assumed to be $50 \%$ in order to take into account the difficulty involved in penetration of the tumor, bioavailability and the specificity of the agent. Differentiating agents may act on more than one layer of the stem cell hierarchy and isolating agents to study the effects of differentiation on one particular layer of cells may not be feasible. The apoptotic rate of stem cells was taken to be zero but this may not necessarily be true for stem cell compartments. This model does not take into account the effect of vasculature and the stromal component of the stem cell niche. It is assumed that the stromal component is unaffected by the perturbing factors. This model is not designed to address the origins of the cancer stem cells and assumes that the entire niche is built from the original cancer stem cell which seeds the compartment. This model does not take into account the possible occurrence of resistance to the perturbing factors. The model assumes that the length of cell cycle is similar for cells in all the layers. The model also assumes that the dedifferentiation rate is similar amongst all the layers in the compartment. Future models will incorporate the effects of stem cell antagonists which act at varying efficiencies ranging from $10-90 \%$ at different time points. The parameters of probability of division, apoptosis, differentiation and dedifferentiation will also be altered over a spectrum to test the formation on new equilibria. The emergence of resistance has not been addressed in this model and will be incorporated into future models.

\section{Conclusion}

The mathematical model of stem cell homeostasis described here serves as a basis for investigating the effect of various perturbing factors. After incorporating minimal probabilities of dedifferentiation at various layers of the stem cell cascade, it becomes evident that differentiating agents which act on specific cell populations are not able to regress the tumor beyond a certain newly established equilibrium. The model also shows that if a differentiating agent acts specifically on the progenitor cell population, it may increase the tumor size and act in a paradoxical manner to drive tumor growth. This effect is a theoretical prediction and is yet to be confirmed experimentally. This model predicts that a dual regimen consisting of a stem cell antagonist and a differentiating agent which acts on multiple cell populations is able to effectively decrease the tumor size and eliminate tumor cells thus preventing recurrence (Table 1 ).

\section{References}

1. Fidler IJ, Kripke ML (1977) Metastasis results from preexisting variant cells within a malignant tumor. Science 197: 893-895.

2. Stenning SP, Parkinson MC, Fisher C, Mead GM, Cook PA, et al. (1998) Postchemotherapy residual masses in germ cell tumor patients: Content, clinical features, and prognosis. Medical research council testicular tumour working party. Cancer 83: 1409-1419.

3. Reya T, Morrison SJ, Clarke MF, Weissman IL (2001) Stem cells, cancer, and cancer stem cells. Nature 414: 105-111.

4. Schatton T, Frank MH (2008) Cancer stem cells and human malignant melanoma. Pigment Cell Melanoma Res 21: 39-55.
5. Singh SK, Hawkins C, Clarke ID, Squire JA, Bayani J, et al. (2004) Identification of human brain tumour initiating cells. Nature 432: 396-401.

6. Al-Hajj M, Wicha MS, Benito-Hernandez A, Morrison SJ, Clarke MF (2003) Prospective identification of tumorigenic breast cancer cells. Proc Natl Acad Sci USA 100: 3983-3988.

7. Li C, Lee CJ, Simeone DM (2009) Identification of human pancreatic cancer stem cells. Methods Mol Biol 568: 161-173.

8. Collins AT, Berry PA, Hyde C, Stower MJ, Maitland NJ (2005) Prospective identification of tumorigenic prostate cancer stem cells. Cancer Res 65: 1094610951.

9. O'Brien CA, Pollett A, Gallinger S, Dick JE (2007) A human colon cancer cel capable of initiating tumour growth in immunodeficient mice. Nature 445: 106110.

10. He X, Marchionni L, Hansel DE, Yu W, Sood A, et al. (2009) Differentiation of a highly tumorigenic basal cell compartment in urothelial carcinoma. Stem Cells 27: 1487-1495.

11. Guddati AK (2012) Ovarian cancer stem cells: Elusive targets for chemotherapy. Medical Oncology 29: 3400-3408.

12. Eppert K, Takenaka K, Lechman ER, Waldron L, Nilsson B, et al. (2011) Stem cell gene expression programs influence clinical outcome in human leukemia. Nat Med 17: 1086-1093.

13. Sarry JE, Murphy K, Perry R, Sanchez PV, Secreto A, et al. (2011) Human acute myelogenous leukemia stem cells are rare and heterogeneous when assayed in NOD/SCID/IL2RYc-deficient mice. J Clin Invest 121: 384-395.

14. Pang R, Law WL, Chu AC, Poon JT, Lam CS, et al. (2010) A subpopulation of cd26+ cancer stem cells with metastatic capacity in human colorectal cancer. Cell stem cell 6: 603-615

15. Hermann PC, Huber SL, Herrler T, Aicher A, Ellwart JW, et al. (2007) Distinct populations of cancer stem cells determine tumor growth and metastatic activity in human pancreatic cancer. Cell stem cell 1: 313-323.

16. Kirkland MA (2004) A phase space model of hemopoiesis and the concept of stem cell renewal. Exp Hematol 32: 511-519.

17. Bao S, Wu Q, McLendon RE, Hao Y, Shi Q, et al. (2006) Glioma stem cells promote radioresistance by preferential activation of the DNA damage response. Nature 444: 756-760.

18. Diehn M, Clarke MF (2006) Cancer stem cells and radiotherapy: New insights into tumor radioresistance. J Natl Cancer Inst 98: 1755-1757.

19. de Thé H, Chen $Z$ (2010) Acute promyelocytic leukaemia: Novel insights into the mechanisms of cure. Nat Rev Cancer 10: 775-783.

20. Piccirillo SG, Reynolds BA, Zanetti N, Lamorte G, Binda E, et al. (2006) Bone morphogenetic proteins inhibit the tumorigenic potential of human brain tumourinitiating cells. Nature 444: 761-765.

21. Williams RT, den Besten W, Sherr CJ (2007) Cytokine-dependent imatinib resistance in mouse bcr-abl+, arf-null lymphoblastic leukemia. Genes Dev 21 2283-2287.

22. Lapidot T, Sirard C, Vormoor J, Murdoch B, Hoang T, et al. (1994) A cell initiating human acute myeloid leukaemia after transplantation into scid mice. Nature 367: 645-668.

23. Quintana E, Shackleton M, Foster HR, Fullen DR, Sabel MS, et al. (2010) Phenotypic heterogeneity among tumorigenic melanoma cells from patients that is reversible and not hierarchically organized. Cancer Cell 18: 510-523.

24. Chaffer CL, Brueckmann I, Scheel C, Kaestli AJ, Wiggins PA, et al. (2011) Normal and neoplastic nonstem cells can spontaneously convert to a stem-like state. Proc Natl Acad Sci USA 108: 7950-7955.

25. Gupta PB, Fillmore CM, Jiang G, Shapira SD, Tao K, et al. (2011) Stochastic state transitions give rise to phenotypic equilibrium in populations of cancer cells. Cell 146: 633-644.

This article was originally published in a special issue, Cancer Stem Cells handled by Editor(s). Fazlul Hoque Sarkar, Wayne State University, USA Asfar Sohail Azmi, Wayne State University, USA 